\title{
Paradoxical Withdrawal of Reflex Vasoconstriction as a Cause of Hemodialysis-induced Hypotension
}

\author{
Richard L. Converse, Jr., Tage N. Jacobsen, Charles M. T. Jost, Robert D. Toto, Paul A. Grayburn, \\ Troy M. Obregon, Fetnat Fouad-Tarazi, * and Ronald G. Victor \\ Department of Internal Medicine, University of Texas Southwestern Medical Center, Dallas, Texas 75235-9034; \\ and ${ }^{*}$ Research Institute of the Cleveland Clinic Foundation, Cleveland, Ohio 44195-5069
}

\begin{abstract}
Acute hypotension is an important complication of hemodialysis, but the underlying mechanisms remain poorly understood. Because hemorrhage-induced hypovolemia can trigger a sudden decrease in sympathetic activity resulting in bradycardia and vasodilation, we hypothesized that hemodialysis-induced hypovolemia also can trigger the same type of vasodepressor reaction, which would exacerbate the volume-dependent fall in blood pressure. We therefore measured blood pressure, vascular resistance, and sympathetic nerve activity (intraneural microelectrodes ) during sessions of maintenance hemodialysis in 7 patients with and 16 patients without a history of hemodialysis-induced hypotension. During hemodialysis, blood pressure at first remained unchanged as calf resistance increased in both hypotension-resistant (from $37 \pm 4$ to $49 \pm 5 \mathrm{U}, P<0.05$ ) and hypotension-prone ( from $42 \pm 6$ to $66 \pm 12 \mathrm{U}, P<0.05$ ) patients; sympathetic activity increased comparably in the subset of patients in whom it could be measured. With continued hemodialysis, calf resistance and sympathetic activity increased further in the hypotension-resistant patients, but in the hypotensionprone patients the precipitous decrease in blood pressure was accompanied by decreases in sympathetic activity, vascular resistance, and heart rate as well as symptoms of vasodepressor syncope. On an interdialysis day, both groups of patients increased vascular resistance normally during unloading of cardiopulmonary baroreceptors with lower body negative pressure and increased heart rate normally during unloading of arterial baroreceptors with infusion of nitroprusside. These findings indicate that in a group of hemodialysis patients without diabetes or other conditions known to impair autonomic reflexes, hemodialysis-induced hypotension is not caused by chronic uremic impairment in arterial or cardiopulmonary baroreflexes but rather by acute, paradoxical withdrawal of sympathetic vasoconstrictor drive producing vasodepressor syncope. (J. Clin. Invest. 1992. 90:1657-1665.) Key words: cardiac afferents • peripheral circulation • shock • sympathetic nervous system
\end{abstract}

\section{Introduction}

Acute hypotension is an important complication of hemodialysis, occurring in up to one-third of chronic dialysis patients

Address reprint requests to Dr. Victor, Cardiology Division, Department of Internal Medicine, University of Texas Southwestern Medical a Center, 5323 Harry Hines Boulevard, Dallas, TX 75235-9034. 1992.

Received for publication 4 March 1992 and in revised form 6 August

J. Clin. Invest.

(C) The American Society for Clinical Investigation, Inc.

$0021-9738 / 92 / 11 / 1657 / 09 \$ 2.00$

Volume 90, November 1992, 1657-1665
(1-4). Although these hypotensive events often require aggressive resuscitative measures and premature termination of hemodialysis, the underlying mechanisms causing this episodic hypotension remain poorly understood.

One hypothesis is that hemodialysis-induced hypotension is a manifestation of uremic autonomic neuropathy, a form of chronic autonomic insufficiency caused by uremic poisoning of the baroreceptors (4-9). Although this hypothesis is widely acclaimed, a causal relationship between impaired baroreflexes and dialysis-induced hypotension has not been established $(10,11)$.

Whereas hypotensive states typically lead to reflex sympathetic activation and vasoconstriction (12), hemorrhagic hypotension can lead to sudden, paradoxical withdrawal of sympathetic drive, causing reflex vasodilation and bradycardia-a vasodepressor reaction that exacerbates the volume-dependent fall in blood pressure (13-15). We therefore hypothesized that the hypovolemia that accompanies hemodialysis also might trigger the abrupt withdrawal of sympathetic vasoconstrictor drive, producing an acute, rather than chronic, form of autonomic insufficiency.

To test this new hypothesis, we measured muscle sympathetic nerve activity (intraneural microelectrodes) and regional vascular resistance during maintenance hemodialysis in patients with and without a history of dialysis-induced hypotension. To test the more traditional hypothesis linking dialysis-induced hypotension to chronic uremic poisoning of baroreceptors, we examined arterial and cardiopulmonary baroreflexes on an interdialysis day in the same patients.

\section{Methods}

\section{Subjects}

The study comprised 23 patients with end-stage renal failure on thriceweekly maintenance hemodialysis of 3-4 h duration and 8 healthy control subjects. Patients were studied at the chronic hemodialysis centers and the human neurophysiology laboratories of Parkland Memorial Hospital, Dallas, TX, and the Cleveland Clinic Foundation, Cleveland, $\mathrm{OH}$. The protocol was approved by the Institutional Review Boards at both facilities and all subjects provided written informed consent before participation.

Based on their blood pressure response during hemodialysis, the patients were prospectively divided into two groups: 7 "hypotensionprone" patients and 16 "hypotension-resistant" patients. Hypotension-prone patients were defined as those in whom a sudden, symptomatic decrease in mean arterial pressure of $>30 \mathrm{mmHg}$ occurred during at least one-third of maintenance hemodialysis sessions. In these patients, dialysis-induced hypotension occurred most predictably during the first dialysis session of the week, when the most volume was removed. These patients were easily identified by the nursing staff and by the patients' own history. Patients classified as "hypotension resistant" rarely, if ever, experienced such episodes.

The etiology of the renal failure included hypertension $(n=9)$, chronic glomerulonephritis $(n=8)$, obstructive uropathy $(n=3)$, 
analgesic-related renal failure $(n=2)$, and Alport's syndrome $(n=1)$. Patients with a history or physical examination indicative of diabetes mellitus, coronary artery disease, congestive heart failure, cardiac arrhythmias, volume overload, alcohol abuse, peripheral neuropathy, or other serious systemic illness were excluded from the study. One-half of our patients were not receiving antihypertensive medications. In those who were receiving antihypertensive medications, these medications were discontinued $48 \mathrm{~h}$ before study, with the exception of five patients in whom it was deemed unsafe to do so. These five patients included two hypotension-prone (taking captopril and metoprolol or captopril, metoprolol, and nifedipine) and three hypotension-resistant (taking captopril and nifedipine, labetolol and nifedipine, or atenolol).

Routine hemodialysis procedures were followed for each patient according to their individual prescriptions. All patients were dialyzed with volumetric control utilizing Fresenius (Feratromics Inc., Concord, CA) model A 20008E machines. Dialysis was performed with cellulose acetate (CDAK 135) dialyzers with blood flow rates ranging from 300 to $400 \mathrm{ml} / \mathrm{min}$, dialysate flow of $500 \mathrm{ml} / \mathrm{min}$, and ultrafiltration rate varying according to prescription. The dialysate composition was as follows: $\mathrm{Na}^{+} 140 \mathrm{meq} /$ liter, $\mathrm{HCO}_{3}^{-} 35 \mathrm{meq} /$ liter, $\mathrm{K}^{+} 2.0 \mathrm{meq} /$ liter, $\mathrm{Cl}^{-} 104 \mathrm{meq} /$ liter, $\mathrm{Ca}^{++} 3.0 \mathrm{meq} /$ liter, $\mathrm{Mg}^{++} 0.75 \mathrm{meq} /$ liter, dextrose $200 \mathrm{mg} / \mathrm{dl}$. Heparinization was achieved with 5,000 units intravenous bolus followed by $500 \mathrm{u} / \mathrm{h}$ intermittent intravenous bolus.

\section{General procedures}

All experiments were performed with the subjects in the supine position. The studies were performed in a quiet area of the hemodialysis center separated from the other patients for the first two protocols and in the human neurophysiology laboratory for the latter three protocols. Heart rate (electrocardiography), blood pressure (Finapres, Ohmeda), and efferent muscle sympathetic nerve activity were recorded continuously; calf blood flow (plethysmography) was recorded once every $15 \mathrm{~s}$, and all data were stored on a TEAC R-71 tape recorder, then converted to a digital signal with Computerscope software (R. C. Electronics, Goleta, CA) and also stored on an IBM AT personal computer. Data were transcribed from FM tape or from computer disk to hard copy using a model ES1000 electrostatic recorder (Gould, Inc., Oxnard, CA).

\section{Recording of sympathetic nerve discharge}

Multiunit recordings of postganglionic sympathetic nerve activity were obtained with unipolar tungsten microelectrodes inserted selectively into muscle nerve fascicles of the peroneal nerve posterior to the fibular head according to the technique of Vallbo et al. (16). Briefly, the neural signals were amplified 20,000-50,000 times, filtered (bandwidth, 700-2,000 Hz), rectified, and integrated (time constant, $0.1 \mathrm{~s}$ ) to obtain a mean voltage display of sympathetic activity. A recording of sympathetic activity was considered acceptable when the neurograms revealed spontaneous, pulse-synchronous bursts of neural activity, with the largest bursts showing a minimal signal-to-noise ratio of 3:1. In each experiment, we documented that we were recording sympathetic outflow to skeletal muscle by demonstrating that the neural activity had no response to arousal stimuli (loud noise or skin pinch) but had a characteristic cardiac rhythmicity (i.e., the interburst interval was equal to or a multiple of the cardiac cycle length).

Sympathetic bursts were detected by inspection of the filtered and mean voltage neurograms. A deflection on the mean voltage display was counted as a burst if it had a minimal signal-to-noise ratio of 2:1. The interobserver and intraobserver variabilities in identifying bursts are $<10 \%$ and $<5 \%$, respectively (17). Inadvertent contraction of the leg muscles adjacent to the recording electrode produces electromyographic artifacts that are easily distinguished from sympathetic bursts; neurograms that revealed such artifacts were excluded from analysis. Nerve traffic was measured as the number of bursts of sympathetic activity per minute, an index of the frequency of sympathetic discharge, and as the burst frequency times mean burst amplitude, an index of total or integrated nerve traffic.

\section{Measurement of regional blood flow}

While recording sympathetic outflow to calf muscles in one leg, we simultaneously measured calf blood flow in the contralateral leg using venous-occlusion plethysmography (18). The calf was elevated above the level of the right atrium to collapse the veins. The circulation to the foot was arrested during the blood-flow determinations, which were performed at 15 -s intervals. Calf vascular resistance was calculated as the mean arterial pressure (one third of the pulse pressure plus diastolic pressure) in millimeters of mercury divided by calf blood flow in milliliters per minute per $100 \mathrm{ml}$ of tissue. The lower body negative pressure protocol (protocol 3) necessitated measuring blood flow in the forearm (outside of the negative pressure chamber) rather than in the calf (which is inside the negative pressure chamber). Forearm blood flow and vascular resistance data were obtained using the same plethysmographic technique.

\section{Echocardiographic measurements of left ventricular dimensions}

Left ventricular end-diastolic and end-systolic dimensions were measured by two-dimensional echocardiography using a parasternal short axis view according to the criteria of the American Society of Echocardiography (19).

\section{Lower body negative pressure}

On the interdialysis day, subjects were brought to the human neurophysiology laboratory and placed in an airtight chamber to the level of the iliac crests. Venous pooling was produced by application of graded lower body negative pressure at -10 and $-20 \mathrm{mmHg}$. The pressure inside of the chamber was measured with a Statham pressure transducer (Gould, Inc.). Lower body negative pressure at -10 and -20 $\mathrm{mmHg}$ is thought to unload primarily low pressure cardiopulmonary (rather than arterial) baroreceptors, since central venous pressure falls but blood pressure, heart rate, and $\mathrm{d} P / \mathrm{d} t$ are unchanged (20).

\section{Determination of plasma norepinephrine and epinephrine concentrations}

We collected samples of blood from the patients from the dialysis access to the forearm fistula ("patient arterial, dialyzer venous"). Samples were collected in prechilled tubes treated with heparin and promptly centrifuged at $4^{\circ} \mathrm{C}$. The samples were assayed by radioenzymatic assay by courtesy of Dr. E. L. Bravo, Cleveland Clinic Foundation. The assay is sensitive to $15 \mathrm{pg} / \mathrm{ml}$.

\section{Experimental protocols}

Protocol 1: responses to conventional hemodialysis. To determine the neurocirculatory adjustments to hemodialysis in patients with and without dialysis-induced hypotension, we recorded blood pressure, heart rate, sympathetic nerve activity, and calf blood flow in 16 hypotension-resistant and 7 hypotension-prone chronic hemodialysis patients during sessions of maintenance hemodialysis and during the reinfusion of blood and normal saline $(300 \mathrm{ml})$ after dialysis. Stable baseline measurements ( $15 \mathrm{~min}$ ) were obtained before the initiation of hemodialysis. In a subset of five hypotension-resistant and five hypotension-prone patients, we performed two dimensional echocardiography and standard 12-lead electrocardiography throughout hemodialysis, paying particular attention to the period shortly preceding the onset of severe hypotension.

To determine whether stimulation of central opiate receptors might contribute to the regulation of sympathetic nerve activity and blood pressure during hemodialysis, we planned to repeat this protocol while infusing the opiate receptor antagonist naloxone $(0.5 \mathrm{mg} / \mathrm{kg})$ during the last third of the hemodialysis session in a subset of our hypotension prone patients. This protocol was performed in only one patient due to its clear lack of efficacy.

Protocol 2: responses to interventional hemodialysis maneuvers. To separate the effects of ultrafiltration from those of dialysis in causing episodic hypotension, in two of the hypotension-resistant and two of 
the hypotension-prone patients we recorded heart rate, blood pressure, and calf blood flow during three separate dialysis maneuvers: $(a)$ the usual hemodialysis procedure (combined ultrafiltration plus dialysis); (b) ultrafiltration alone; and $(c)$ dialysis alone.

Protocol 3: responses to lower body negative pressure. In four hypotension-prone and four hypotension-resistant hemodialysis patients and eight healthy control subjects, we measured blood pressure, heart rate, and forearm blood flow during unloading of mainly cardiopulmonary baroreceptors with graded lower body negative pressure at -10 and $-20 \mathrm{mmHg}$ for $2 \mathrm{~min}$ at each consecutive level.

Protocol 4: responses to intravenous nitroprusside. In four hypotension-prone and four hypotension-resistant hemodialysis patients and eight healthy control subjects, we measured increases in heart rate produced by nitroprusside-induced decreases in mean arterial pressure of -10 and $-20 \mathrm{mmHg}$ for $2 \mathrm{~min}$ at each consecutive dose. In each subject, arterial baroreflex gain was calculated as the increase in heart rate (beats/minute) per unit decrease in mean arterial pressure $(\mathrm{mmHg})$.

Protocol 5: responses to the Valsalva maneuver. In a subset of seven hypotension-resistant and one hypotension-prone hemodialysis patients, we compared responses of heart rate and muscle sympathetic nerve activity during performance of the Valsalva maneuver, which has been the standard test to assess baroreflex function in hemodialysis patients. This maneuver was performed by instructing subjects to exhale into a pressure manometer for $10 \mathrm{~s}$. Subjects were instructed to perform only a mild expiratory effort so as not cause inadvertent contraction of the leg muscles which would dislodge the microelectrode. In each patient, we measured the peak decrease in cardiac cycle length and increase in muscle sympathetic activity during phases II and III (strain) and the peak increase in cardiac cycle length and decrease in muscle sympathetic activity during phase IV (release). Because the Valsalva maneuver perturbs multiple reflex mechanisms, no attempt was made to compare responses between subject groups.

\section{Statistical analysis}

Statistical analysis was performed using Student's unpaired t-test to compare baseline parameters between the two groups of dialysis patients. Group differences in responses during hemodialysis and during lower body negative pressure were compared using repeated measures analysis of variance with Dunnett's post hoc test for multiple comparisons. A $P$ value of $<0.05$ was considered to be statistically significant. Results are expressed as means $\pm \mathrm{SE}$.

\section{Results}

The clinical characteristics and baseline values of the hemodialysis patients are shown in Table I. There were no statistical differences between hypotension-resistant and hypotensionprone patients.

Recordings of baseline muscle sympathetic nerve activity were obtained in 16 of the 23 hemodialysis patients. Owing to inadvertent leg movements ("restless legs syndrome") (21), stable recordings of sympathetic activity could be maintained for a substantial portion of the hemodialysis session in only six of these patients: in two hypotension-prone patients, sympathetic activity was recorded during almost the entire hemodialysis session, including the episodes of acute hypotension; in one hypotension-resistant patient, sympathetic activity was recorded during the entire hemodialysis session, including reinfusion of blood after hemodialysis; in two additional hypotension-resistant patients, sympathetic activity was recorded during the first third of the hemodialysis procedure, before the recordings deteriorated; and in a fourth hypotension-resistant patient, we could obtain an adequate recording of sympathetic activity only during the last third of hemodialysis and main-
Table I. Baseline Characteristics of Patients Undergoing Maintenance Hemodialysis

\begin{tabular}{|c|c|c|}
\hline & $\begin{array}{l}\text { Hypotension-resistant } \\
\text { patients }(n=16)\end{array}$ & $\begin{array}{l}\text { Hypotension-prone } \\
\text { patients }(n=7)\end{array}$ \\
\hline Age, $y r$ & $43 \pm 3$ & $51 \pm 5$ \\
\hline Predialysis weight, $\mathrm{kg}$ & $80 \pm 6$ & $79 \pm 6$ \\
\hline $\begin{array}{l}\text { Weight change with } \\
\text { dialysis, } k g\end{array}$ & $-3.3 \pm 0.4$ & $-3.5 \pm 0.6$ \\
\hline $\begin{array}{l}\text { Percentage of pre-dialysis } \\
\text { weight }\end{array}$ & $-4.3 \pm 0.5$ & $-4.3 \pm 0.6$ \\
\hline Sodium, mmol/liter & $137 \pm 1$ & $139 \pm 1$ \\
\hline Potassium, $\mathrm{mmol} /$ liter & $5.0 \pm 0.2$ & $5.1 \pm 0.3$ \\
\hline Carbon dioxide, $\mathrm{mmol} / \mathrm{liter}$ & $22 \pm 1$ & $21 \pm 1$ \\
\hline Urea nitrogen, $\mathrm{mmol} / \mathrm{liter}$ & $25 \pm 3$ & $24 \pm 4$ \\
\hline Creatinine, $\mu \mathrm{mol} / \mathrm{liter}$ & $1410 \pm 120$ & $1620 \pm 110$ \\
\hline Hematocrit, \% & $27 \pm 1$ & $29 \pm 2$ \\
\hline Norepinephrine, $p g / m l$ & $503 \pm 98$ & $436 \pm 102$ \\
\hline Heart rate, beats $/ \mathrm{min}$ & $74 \pm 4$ & $71 \pm 4$ \\
\hline $\begin{array}{l}\text { Mean arterial pressure, } \\
\quad \mathrm{mmHg}\end{array}$ & $105 \pm 5$ & $116 \pm 6$ \\
\hline $\begin{array}{l}\text { Calf blood flow, } \\
\mathrm{ml} / \mathrm{min} \text { per } 100 \mathrm{ml}\end{array}$ & $3.3 \pm 0.4$ & $3.0 \pm 0.3$ \\
\hline $\begin{array}{l}\left(\text { dyn } \cdot s \cdot \mathrm{min}^{-5}\right) \\
\text { Muscle sympathetic nerve }\end{array}$ & $37 \pm 4$ & $42 \pm 6$ \\
\hline activity, bursts/min & $56 \pm 4$ & $59 \pm 5$ \\
\hline
\end{tabular}

Data are mean \pm SE.

tained that recording during the period of volume reinfusion. Sympathetic activity was also recorded during performance of the Valsalva maneuver in eight patients and during spontaneous extrasystoles in nine patients.

Responses to hemodialysis. During the first two-thirds of hemodialysis, in both hypotension-resistant and hypotensionprone patients arterial pressure was well maintained as calf vascular resistance and sympathetic activity increased progressively, with little change in heart rate (Table II and Fig. 1). For comparable decreases in body weight during hemodialysis, calf vascular resistance increased comparably in the two groups. During the last third of hemodialysis, however, the neurocirculatory responses differed markedly in the two groups. In the hypotension-resistant patients, vascular resistance and sympathetic activity continued to increase throughout the last third of hemodialysis (Table II and Fig. 1). During reinfusion of $\sim 300$ $\mathrm{ml}$ of blood and saline after hemodialysis, calf resistance decreased promptly from $58 \pm 6$ to $45 \pm 5 \mathrm{U}(P<0.05)$; sympathetic activity decreased proportionately in the two patients in whom it was measured.

In the hypotension-prone patients, however, mean arterial pressure decreased by $17 \pm 7 \mathrm{mmHg}$ and heart rate increased by $15 \pm 6$ beats $/ \mathrm{min}(P<0.05$ vs. baseline $)$ shortly before the precipitous fall in blood pressure. As mean arterial pressure subsequently fell abruptly from $99 \pm 5$ to $60 \pm 6 \mathrm{mmHg}$, sympathetic nerve activity, calf vascular resistance, and heart rate did not increase further but rather fell precipitously to baseline values (Table II and Fig. 1) as typical vasovagal symptoms developed in all of these patients. In one of these patients, these responses and symptoms were not attenuated and seemed to be exacer- 


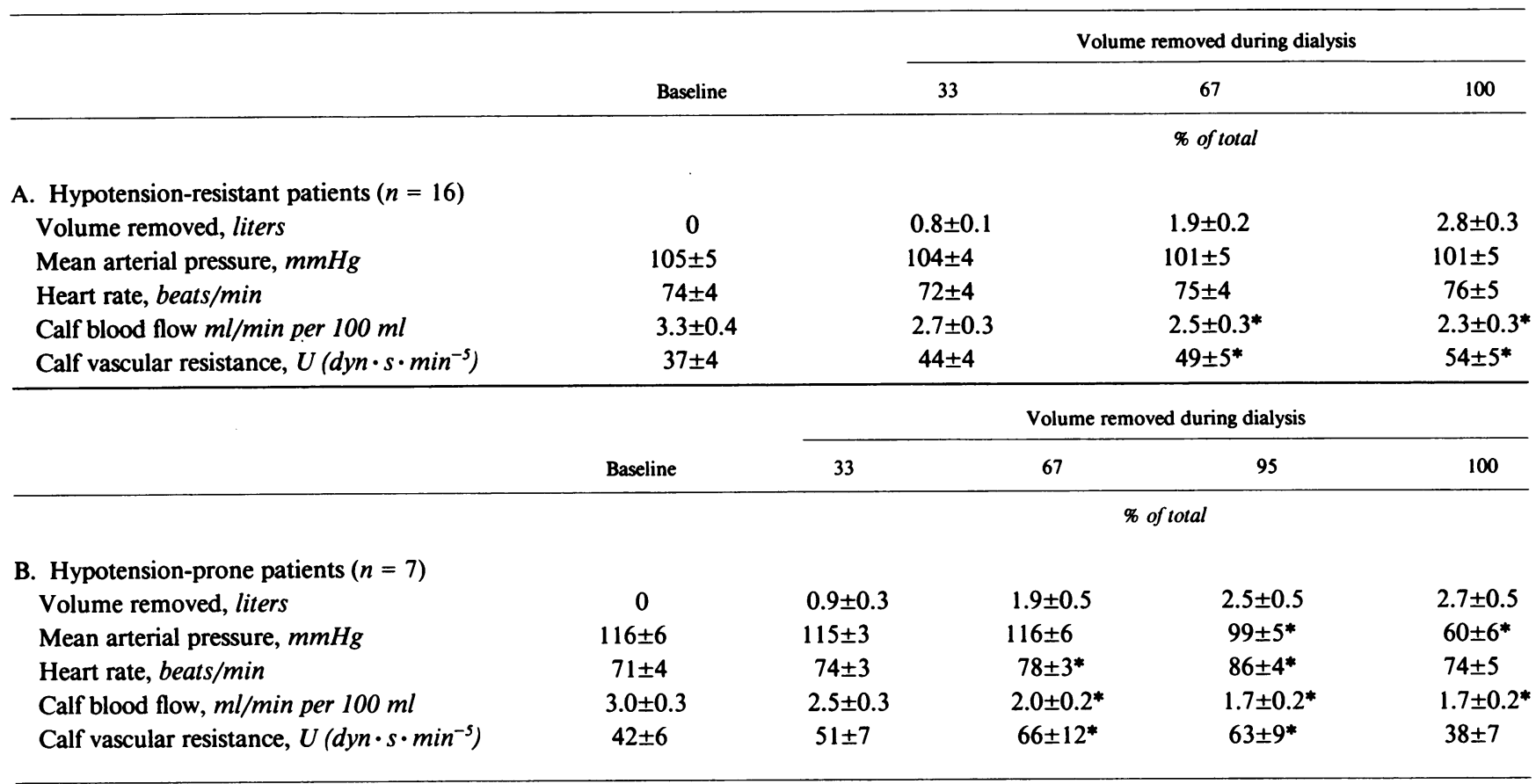

Data are mean \pm SE. $* P<0.05$ vs. baseline.

bated by intravenous infusion of the opiate receptor antagonist naloxone.

In the patients with prolonged sympathetic nerve recordings, changes in sympathetic activity closely paralleled the changes in calf vascular resistance during $(a)$ nonhypotensive hemodialysis, $(b)$ hemodialysis-induced hypotension, and $(c)$ reinfusion of blood after hemodialysis (Fig. 2).

Plasma norepinephrine did not change significantly from the start to the end of hemodialysis in either hypotension-resistant patients (from $613 \pm 117$ to $657 \pm 140 \mathrm{pg} / \mathrm{ml}$ ) or in hypotension-prone patients (from $525 \pm 68$ to $591 \pm 23 \mathrm{pg} / \mathrm{ml}$ ). Plasma epinephrine was unchanged during hemodialysis in the hypotension-resistant patients (from $83 \pm 18$ to $96 \pm 53 \mathrm{pg} / \mathrm{ml}$ ) but increased during hemodialysis-induced hypotensive episodes in the three patients in whom it was measured (from $52 \pm 9$ to $143 \pm 53 \mathrm{pg} / \mathrm{ml}$ ).

In the hypotension-prone patients, hemodialysis was accompanied by progressive reductions in left ventricular endsystolic dimension, which approached cavity obliteration before the onset of the hypotensive episodes (Fig. 3 and Table III). In the hypotension-resistant patients, however, hemodialysis was not accompanied by significant changes in left ventricular dimensions.

In both groups of patients, hemodialysis produced no echocardiographic or electrocardiographic evidence of myocardial ischemia, even during the hypotensive episodes.

Fig. 4 shows the calf vascular responses to three different hemodialysis maneuvers. Ultrafiltration alone, without dialysis, reproduced both the increases in calf resistance that accompany nonhypotensive hemodialysis and the sudden decreases in calf resistance that accompany hemodialysis-induced hypotension. In contrast, dialysis alone, without ultrafiltration, had no effect on calf vascular resistance.
Responses to tests of baroreflex function. Normal baroreflex control of muscle sympathetic nerve activity was demonstrated by the observations that sympathetic activity: $(a)$ exhibited normal cardiac rhythmicity (i.e., the minimum interburst interval equals the cardiac cycle length) in each of the 16 patients in whom it was measured (Fig. 5); (b) increased abruptly with the sharp fall in blood pressure produced by spontaneous extrasystoles (nine patients) (Figs. 5 and 6 ), and (c) increased normally during phases II and III of the Valsalva maneuver and decreased upon its release (phase IV) (Fig. 6). During phases II and III of the Valsalva maneuver, muscle sympathetic activity increased from $782 \pm 100$ to $2,302 \pm 330 \mathrm{U}(P<0.05)$ while cardiac cycle length decreased from $794 \pm 36$ to $700 \pm 31 \mathrm{~ms}(P$ $<0.05$ ), while during phase IV, muscle sympathetic activity decreased to $425 \pm 88 \mathrm{U}$ ( $P<0.05$ vs. baseline) and cardiac cycle length returned to baseline ( $834 \pm 44 \mathrm{~ms}$ ).

Forearm vascular resistance increased normally during unloading of cardiopulmonary baroreceptors with graded lower body negative pressure in both hypotension-prone and -resistant hemodialysis patients (Table IV).

Heart rate increased normally in both groups of patients during unloading of arterial baroreceptors with graded intravenous infusion of nitroprusside (Fig. 7).

\section{Discussion}

The data in this study provide the first direct measurements of sympathetic nerve activity and regional vascular resistance during hemodialysis. The principal new conclusion is that, in a group of hemodialysis patients without diabetes mellitus or other conditions known to impair autonomic reflexes, hemodialysis-induced hypotension is not caused by a chronic uremic impairment of arterial or cardiopulmonary baroreflexes but 


\section{Hypotension-Resistant Patient}

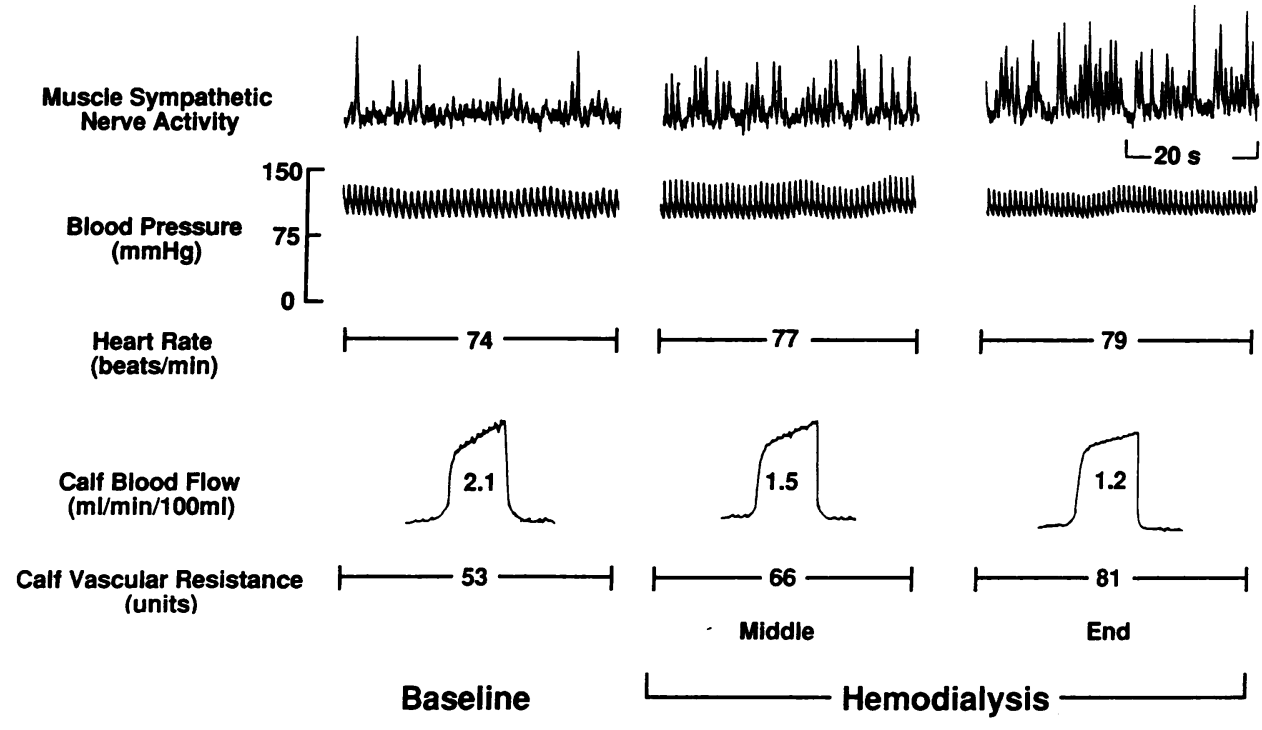

\section{Hypotension-Prone Patient}

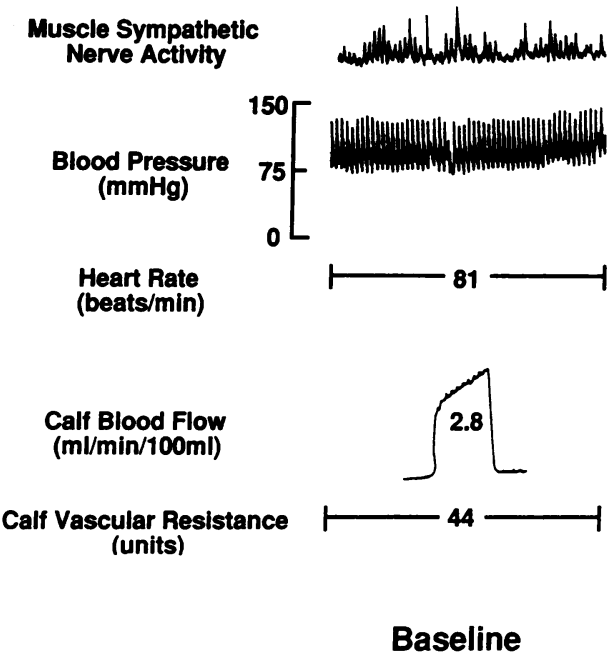

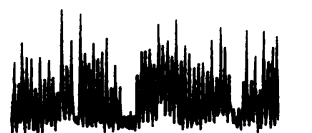
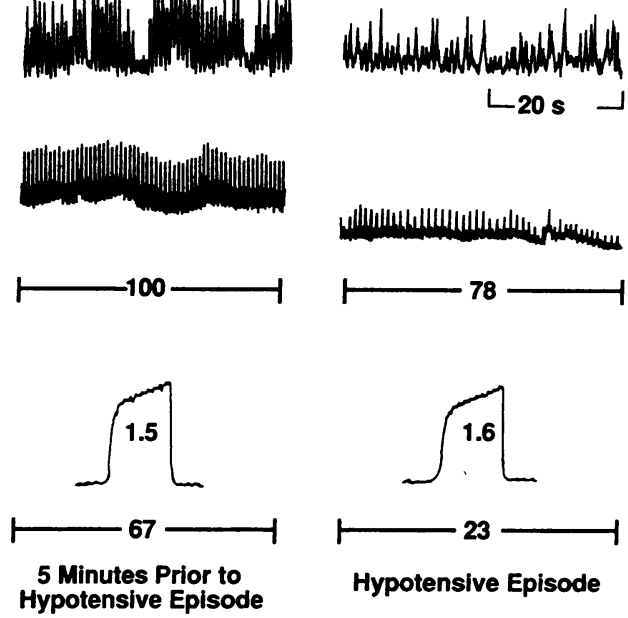

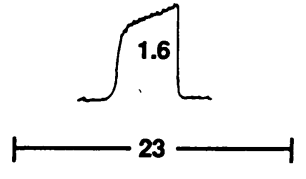

Hypotensive Episode
Figure 1. Segments of the original records from (top) one hypotension-resistant patient and (bottom) one hypotension-prone patient showing the mean voltage display of muscle sympathetic nerve activity, the blood pressure tracing, and plethysmographic tracings of calf blood flow at baseline and during hemodialysis. (Top) In the hypotension-resistant patient, hemodialysis caused progressive increases in sympathetic nerve activity and corresponding decreases in calf blood flow while blood pressure and heart rate remained stable. (Bottom) In the hypotension-prone patient, hemodialysis initially caused similar increases in sympathetic nerve activity and decreases in calf blood flow to those seen in the hypotension-resistant patient but these responses were accompanied by moderate decreases in blood pressure and increases in heart rate. With further hemodialysis, a precipitous fall in blood pressure was accompanied by precipitous decreases in sympathetic activity, heart rate, and vascular resistance. rather by an acute, paradoxical withdrawal of sympathetic vasoconstrictor drive producing vasodepressor syncope. Our findings, however, do not exclude the possibility that in other groups of hemodialysis patients, such as those with diabetic autonomic neuropathy, impaired baroreflexes indeed may contribute to hypotension during hemodialysis.

In addition to patient selection criteria, which were more narrowly defined in this than in most previous studies, there are other important methodologic differences between our study and the previous studies suggesting that chronic uremic impairment of the afferent arm of the arterial baroreflex is an important cause of hemodialysis-induced hypotension (4-9). First, whereas previous conclusions were derived mainly from nonspecific, semiquantitative autonomic function tests, the use of more specific and quantifiable reflex maneuvers allowed us to conclude that chronic uremia per se does not attenuate a number of specific reflexes, including arterial baroreflex control of heart rate and cardiopulmonary baroreflex control of vascular resistance. Second, we performed our neurocirculatory studies during actual sessions of hemodialysis as well as during the interdialysis period, allowing us to separate the autonomic effects of chronic uremia (which were undetectable) from those of acute hemodialysis (which were profound).

An important feature of this study is the demonstration of surprisingly normal baroreflex function in patients with chronic uremia, even in those with a history of dialysis-induced hypotension. This interpretation, which is consistent with and extends recent observations by Nakashima et al. (11), is based upon multiple lines of evidence. Heart rate, for example, increased normally during decreases in blood pressure produced by nitroprusside, documenting normal arterial baroreflex control of heart rate in hypotension-prone as well as in hypotension-resistant dialysis patients. In the hypotension-prone patients, the arterial baroreflex also functioned normally during hemodialysis since, before the onset of severe hypotension, a moderate decrease in arterial pressure was accompanied by a 


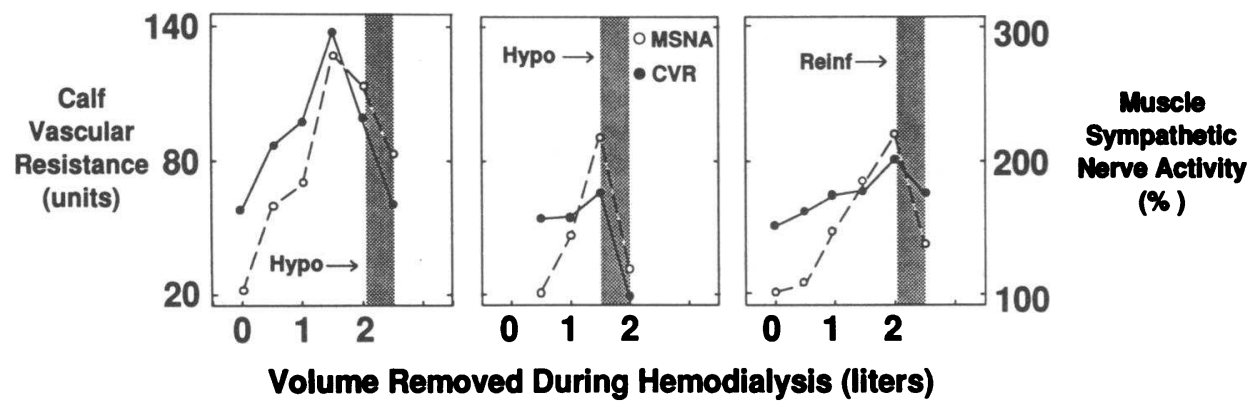

Figure 2. Individual data from three patients showing changes in calf vascular resistance $(C V R)$ and the corresponding changes in muscle sympathetic nerve activity ( $M S N A$ ) during hemodialysis (all three panels) and during reinfusion of blood and saline immediately after hemodialysis (right panel only). In the left and middle panels, the shaded area delineates the sudden episodes of hypotension ( $\mathrm{Hypo}$ ); in the right panel, the shaded area delineates the rapid reinfusion ( Reinf) of blood and saline after hemodialysis. Sympathetic activity and vascular resistance showed parallel responses during nonhypotensive hemodialysis, during hemodialysis-induced hypotension, and during reinfusion of blood after hemodialysis.

proportionate increase in heart rate. However, recent data suggest that preservation of baroreflex control of heart rate cannot necessarily be taken to indicate preservation of baroreflex control of peripheral sympathetic nerve activity and vascular resistance (22), which is more important than heart rate in the reflex regulation of arterial pressure. We subsequently addressed this issue using microelectrode recordings of muscle sympathetic nerve activity together with corresponding measurements of regional vascular resistance.

Because the "restless legs syndrome" (21) was present in most of our patients, we were unable to calculate the mean

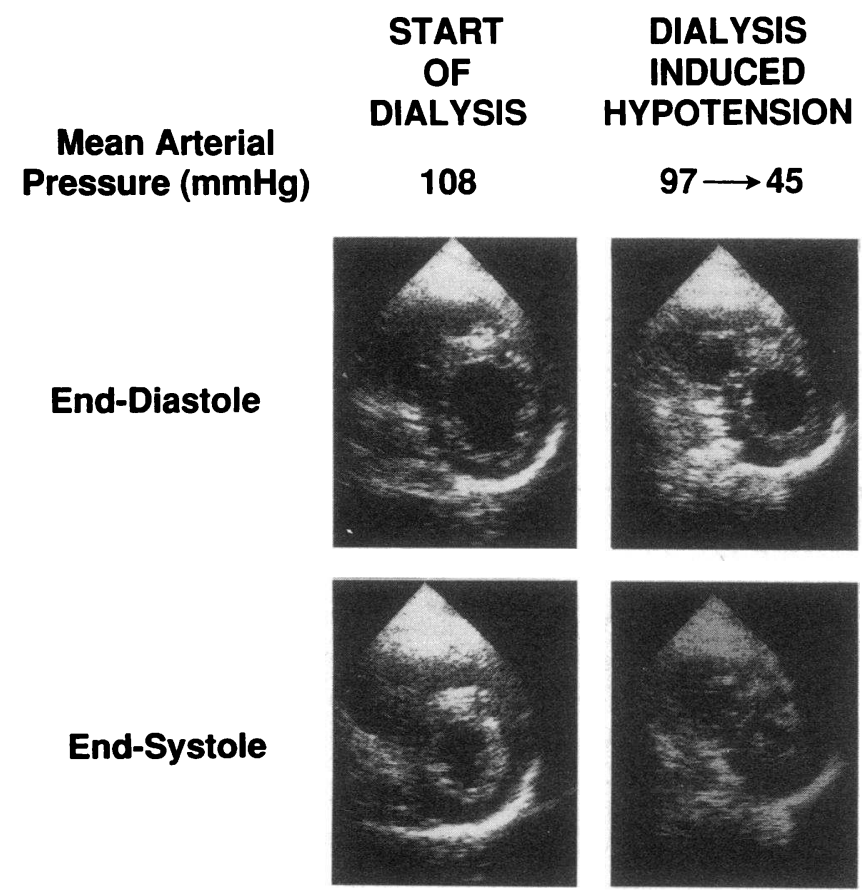

Figure 3. Illustrative parasternal short-axis two-dimensional echocardiographic left ventricular end-diastolic and end-systolic frames from a hypotension-prone hemodialysis patient taken at the start of hemodialysis (left frames) and immediately prior to the onset of an acute hypotensive episode (right frames). The corresponding values of mean arterial pressure are shown above the echocardiographic frames. In this patient, the onset of hemodialysis-induced hypotension was preceded by a small decrease in end-diastolic dimension and a marked decrease in end-systolic dimension, the latter approaching left ventricular cavity obliteration. increase in sympathetic nerve activity evoked by a given decrease in arterial pressure (i.e., baroreflex gain) and therefore cannot exclude the possibility that chronic uremia and a history of dialysis-induced hypotension might be accompanied by a subtle attenuation of arterial baroreflex control of muscle sympathetic nerve activity. However, the findings that the sympathetic activity not only retained its normal cardiac rhythmicity in all of our dialysis patients but also increased with each decrease in blood pressure evoked by spontaneous extrasystoles and increased markedly with the Valsalva maneuver exclude the possibility of complete sinoaortic denervation (23, 24 ) and support the conclusion that a major impairment in baroreflex control of sympathetic nerve activity is not a necessary consequence of chronic uremia.

The lack of an increase in heart rate during nonhypotensive hemodialysis should not be interpreted as indicating a selective impairment in the baroreflex regulation of sympathetic outflow to the heart. During nonhypotensive hemorrhage (simulated by nonhypotensive lower body negative pressure), an increase in peripheral vascular resistance without an increase in heart rate is the expected pattern of reflex response to unloading of mainly "low pressure" cardiopulmonary, rather than "high pressure" arterial, baroreceptors (20).

In the subset of patients in whom sympathetic nerve activity was recorded continuously throughout most of the hemodialysis period, the parallel changes in sympathetic activity and

Table III. Echocardiographic Changes in Left Ventricular Dimensions during Hemodialysis

\begin{tabular}{lccc}
\hline & \multicolumn{3}{c}{ Hemodialysis } \\
\cline { 2 - 4 } & Start & Middle & End \\
\hline $\begin{array}{c}\text { A. End-diastolic dimension, } c m \\
\text { Hypotension-resistant } \\
\text { patients }(n=5)\end{array}$ & & & \\
$\begin{array}{c}\text { Hypotension-prone } \\
\text { patients }(n=5)\end{array}$ & $5.2 \pm 0.3$ & $4.7 \pm 0.2$ & $4.8 \pm 0.2$ \\
$\begin{array}{c}\text { B. End-systolic dimension, } c m \\
\text { Hypotension-resistant } \\
\text { patients }(n=5)\end{array}$ & $4.2 \pm 0.3$ & $3.9 \pm 0.2$ & $3.7 \pm 0.2$ \\
$\begin{array}{c}\text { Hypotension-prone } \\
\text { patients }(n=5)\end{array}$ & $2.8 \pm 0.1$ & $2.8 \pm 0.1$ & $2.7 \pm 0.1$ \\
& $2.8 \pm 0.1$ & $2.6 \pm 0.1$ & $2.2 \pm 0.2 *$
\end{tabular}

Data are mean \pm SE. ${ }^{*} P<0.05$ vs. start of hemodialysis. 


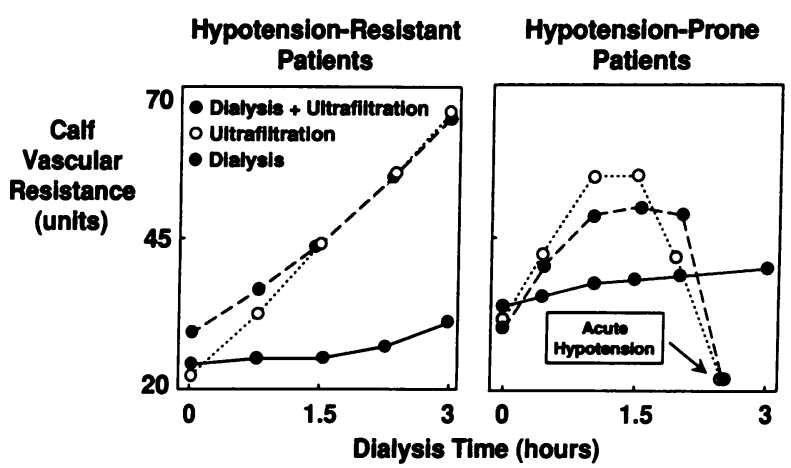

Figure 4. Pooled data from two hypotension-prone and two hypotension-resistant hemodialysis patients plotting changes in calf vascular resistance as a function of the time during each of three separate hemodialysis maneuvers: the usual hemodialysis procedure (ultrafiltration plus dialysis), dialysis alone without ultrafiltration, and ultrafiltration alone without dialysis. In both the hypotension-resistant and hypotension-prone patients, ultrafiltration alone reproduced the vascular responses caused by the usual hemodialysis procedure. In contrast, dialysis alone had no effect on vascular resistance in these patients.

regional vascular resistance during nonhypotensive hemodialysis, during hemodialysis-induced hypotension, and during reinfusion of blood after hemodialysis suggest that chronic uremia does not impair the ability of the peripheral vasculature to respond appropriately to baroreflex changes in sympathetic neural activity. This interpretation is strengthened by the additional finding that in both hypotension-prone and -resistant patients regional vascular resistance also increased normally during unloading of cardiopulmonary baroreceptors with graded lower body negative pressure. From these findings, it would be very difficult to explain dialysis-induced hypotension in our patients on the basis of chronic uremic poisoning of either arterial or cardiopulmonary baroreceptors.

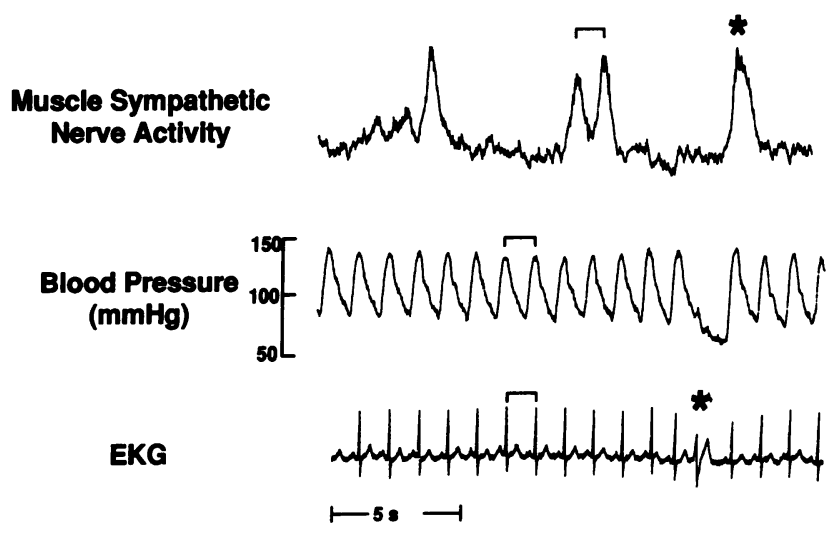

Figure 5. An illustrative record from a hypotension-prone hemodialysis patient showing muscle sympathetic nerve activity (mean voltage neurogram), the blood pressure tracing, and the electrocardiogram recorded at a rapid paper speed to illustrate the temporal relationships. In this patient, muscle sympathetic nerve activity is under baroreflex regulation because the bursts of sympathetic activity are locked to the cardiac cycle (the minimum interburst interval equals the cardiac cycle length) and a decrease in blood pressure after a spontaneous extrasystole $\left({ }^{*}\right)$ triggers a large burst of sympathetic activity.

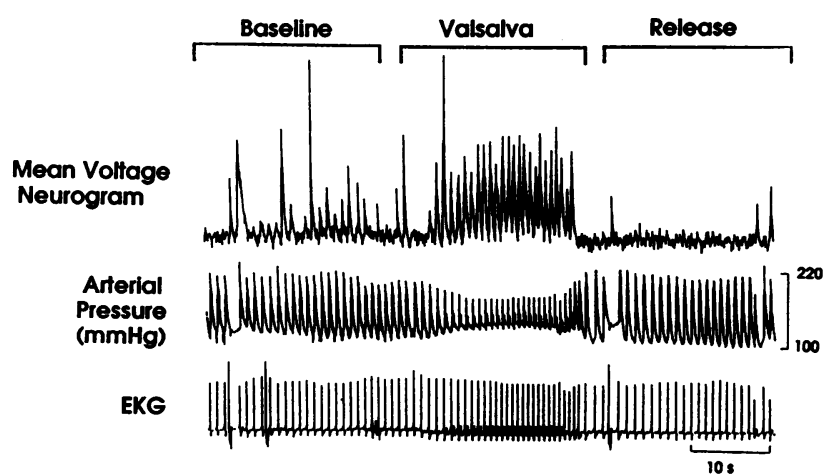

Figure 6. An illustrative record from another hypotension-prone hemodialysis patient showing changes in muscle sympathetic nerve activity (mean voltage neurogram), arterial pressure, and the electrocardiogram produced by performance of the Valsalva maneuver and by spontaneous extrasystoles. The evidence for normal baroreflex function in this patient is that sympathetic activity $(a)$ increases normally during the Valsalva maneuver and decreases normally upon its release, and $(b)$ increases abruptly with each extrasystole that causes a sharp fall in blood pressure.

Indeed, during the mild decreases in blood pressure observed before the onset of frank dialysis-induced hypotension, the progressive increases in heart rate, vascular resistance, and efferent sympathetic activity were most likely caused by a progressive reduction in inhibitory afferent baroreceptor input to the vasomotor center. If uremic poisoning of these afferent nerves had caused baroreceptor activity to be substantially reduced under basal conditions and thus could not decrease much further during hemodialysis-induced hypotension, it follows that efferent sympathetic nerve activity should have in-

Table IV. Hemodynamic Responses to Graded Lower Body Negative Pressure (LBNP)

\begin{tabular}{|c|c|c|c|}
\hline & Baseline & LBNP-10 & LBNP-20 \\
\hline \multicolumn{4}{|l|}{ Mean arterial pressure, $\mathrm{mmHg}$} \\
\hline Hypotension-resistant patients & $96 \pm 5$ & $98 \pm 6$ & $97 \pm 6$ \\
\hline Hypotension-prone patients & $110 \pm 8$ & $110 \pm 7$ & $112 \pm 8$ \\
\hline Control subjects & $86 \pm 2$ & $82 \pm 1$ & $81 \pm 2$ \\
\hline \multicolumn{4}{|l|}{ Heart rate, beats/min } \\
\hline Hypotension-resistant patients & $72 \pm 6$ & $72 \pm 7$ & $74 \pm 7$ \\
\hline Hypotension-prone patients & $77 \pm 3$ & $78 \pm 2$ & $78 \pm 2$ \\
\hline Control patients & $61 \pm 3$ & $60 \pm 2$ & $63 \pm 3$ \\
\hline \multicolumn{4}{|l|}{$\begin{array}{l}\text { Forearm blood flow, } \\
\mathrm{ml} / \mathrm{min} \text { per } 100 \mathrm{ml}\end{array}$} \\
\hline Hypotension-resistant patients & $5.0 \pm 1.7$ & $4.1 \pm 1.4$ & $3.6 \pm 1.2^{*}$ \\
\hline Hypotension-prone patients & $4.0 \pm 0.6$ & $3.6 \pm 0.7$ & $3.2 \pm 0.6$ \\
\hline Control patients & $4.2 \pm 0.8$ & $3.8 \pm 0.7$ & $3.1 \pm 0.6^{*}$ \\
\hline \multicolumn{4}{|l|}{$\begin{array}{c}\text { Forearm vascular resistance, } \\
\qquad\left(d y n \cdot s \cdot \mathrm{min}^{-5}\right)\end{array}$} \\
\hline Hypotension-resistant patients & $28 \pm 7$ & $33 \pm 6$ & $37 \pm 8^{*}$ \\
\hline Hypotension-prone patients & $33 \pm 9$ & $39 \pm 11$ & $43 \pm 13^{*}$ \\
\hline Control subjects & $25 \pm 4$ & $27 \pm 4$ & $34 \pm 6^{*}$ \\
\hline
\end{tabular}

Data are mean \pm SE for five hypotension-resistant patients, five hypotension-prone patients, and nine control subjects. ${ }^{*} P<0.05$ vs. baseline. 


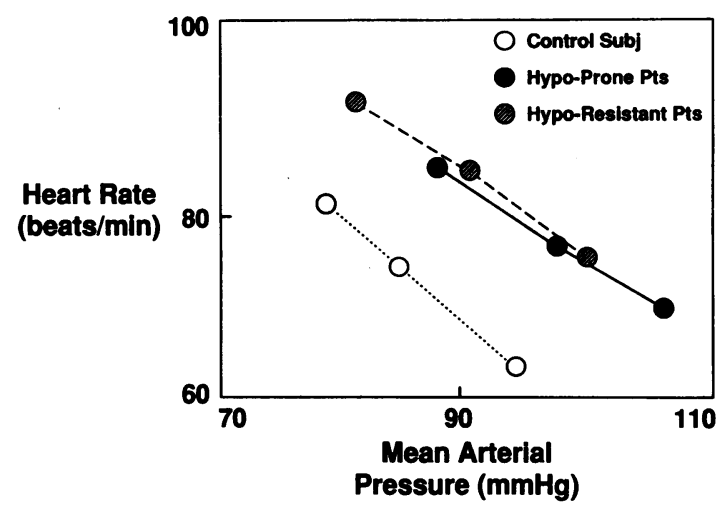

Figure 7. Mean baroreflex curves for four hypotension-resistant hemodialysis patients, four hypotension-prone patients, and eight normotensive control subjects showing baroreflex-mediated increases in heart rate produced by nitroprusside-induced graded reductions in mean arterial pressure. The slopes and correlation coefficients for the individual baroreflex curves are: slope $=-1.1 \pm 0.4, r=0.99 \pm 0.01$ for hypotension-prone patients; slope $=-0.8 \pm 0.1, r=0.99 \pm 0.01$ for hypotension-resistant patients; and slope $=-1.1 \pm 0.2, r=0.97 \pm 0.02$ for control subjects. Although the blood pressures are higher in the patients than controls, the slopes of the baroreflex curves are equivalent in all three groups.

creased to its maximal value in the initial phase of hemodialysis and thus would have been unable to increase further during the subsequent severe hypotensive episodes. Because sympathetic activity, heart rate, and vascular resistance did not remain elevated but rather decreased during dialysis-induced hypotension, these findings suggest a sudden increase, not a decrease (or "poisoning"), in baroreceptor function and demonstrate that this episodic hypotension is a form of vasodepressor syncope. This characteristic pattern of autonomic response was accompanied by the classic premonitory symptoms of vasodepressor syncope-nausea, yawning, diaphoresis, and giddiness -as well as the characteristic responses of plasma catecholamines-large increases in plasma epinephrine with no significant change in plasma norepinephrine (25).

Our experiments in which we divided the usual hemodialysis procedure (dialysis plus ultrafiltration) into its component parts (dialysis alone and ultrafiltration alone) strongly suggest that hypovolemia per se, rather than changes in the uremic milieu (e.g., ion shifts which might be expected to alter baroreceptor discharge ) (26), is the primary stimulus that triggers this vasodepressor reaction. This interpretation is consistent with an increasing body of experimental evidence that the abrupt withdrawal of sympathetic activation, with bradycardia and vasodilation, is a predictable autonomic response to hypovolemic hypotension (13-15). In humans, hypotension with inappropriately normal or frankly decreased heart rate previously has been observed during hemorrhagic shock (27), infusion of catecholamines during upright tilt (28), and infusion of vasodilators, the latter being accompanied by a parallel decrease in muscle sympathetic nerve activity $(29,30)$.

Although the precise underlying mechanisms causing hypovolemia-induced vasodepressor syncope are incompletely understood, the present data provide some important clues about the conditions required to trigger this reaction during hemodialysis. Such vasodepressor reactions have been attributed both to central and to reflex inhibition of sympathetic outflow. Emotional fainting (31), causing inhibition of central sympathetic outflow, is unlikely to explain hemodialysis-induced hypotension. None of our patients had a history of emotional fainting outside of the dialysis unit and none could identify any painful or emotional factor that precipitated the episodes of acute hypotension, many of which awakened the patients from sleep. Activation of opiate receptors also has been implicated in causing central inhibition of sympathetic outflow during hypovolemic hypotension $(32,33)$. Although opiate receptor blockade with naloxone has been shown to reverse hemorrhage-induced sympathoinhibition in conscious rabbits $(32,33)$, it has not proven to be effective in treating hemorrhagic shock in humans (34). Naloxone does not appear to prevent vasodepressor syncope during simulated orthostatic stress (35), and in one of our patients did not prevent and may have exacerbated the acute hypotensive episode during hemodialysis.

The most widely accepted theory is that hypovolemia-induced vasodepressor syncope is caused by activation of cardiac afferents, located primarily in the inferoposterior wall of the left ventricle, triggering reflex inhibition of sympathetic outflow $(36,37)$. Because of the high incidence of coronary artery disease in chronic hemodialysis patients $(4,38)$, we considered the possibility that during hemodialysis cardiac afferents and reflex inhibition of sympathetic outflow might be activated by acute myocardial ischemia (39). In our patients, however, this is an unlikely explanation for vasodepressor syncope because the hypotensive episodes were not accompanied by any electrocardiographic or echocardiographic evidence of myocardial ischemia.

Studies in laboratory animals have advanced the theory that during hypovolemic hypotension ventricular mechanoreceptor afferents are activated "paradoxically" by excessive deformation of their receptive fields when the adrenergically stimulated heart contracts forcefully around an almost empty ventricular chamber $(36,37)$. Previous echocardiographic studies in patients have demonstrated that hemodialysis causes both decreased ventricular chamber size and increased ventricular contractility (40), which are the necessary conditions for triggering paradoxical activation of ventricular mechanoreceptor afferents. Our echocardiographic demonstration that a marked reduction in left ventricular end-systolic dimension (approaching cavity obliteration) during hemodialysis preceded, rather than followed, the onset of vasodepressor syncope is consistent with this ventricular afferent hypothesis. However, multiple, redundant, and as yet unidentified mechanisms also may be involved (14, 30, 41-43).

The present data do not provide a definitive explanation for the observation that a paradoxical decrease in sympathetic activity is much more common in some dialysis patients than in others. However, based on the sympathetic nerve responses during hemorrhagic hypotension in experimental animals $(13-15,33)$, we speculate that this type of vasodepressor reaction could be triggered in any individual if sufficient intravascular volume were removed.

In summary, this study demonstrates that baroreflex dysfunction is not a necessary consequence of chronic uremia and is not a necessary condition for hemodialysis-induced hypotension. The principle new concept arising from this work is that abrupt withdrawal of reflex vasoconstriction is one important cause of hypotension during hemodialysis. 


\section{Acknowledgments}

The authors are indebted to Ms. Patricia Powell for expert secretarial assistance; Rich Cooley, LeAnn Ermel, Christine Bennett, and Jay Cology for superb research assistance; Drs. R. Sanders Williams, Robert Graham, Jere H. Mitchell, Donald W. Seldin, Lee Henderson, and the members of the Medical Advisory Board of the Extramural Grant Program, Baxter Healthcare Corporation (Renal Division) for their continued support and critical review of this work; Dr. William L. Henrich for his helpful comments and critical reading of this manuscript; Drs. Magnus O. Magnusson, Robert J. Heyka, Frank Cosentino, and Emil P. Paganini for allowing us to study their patients at the Cleveland Clinic Foundation; and Dr. E. L. Bravo for performing the plasma catecholamines assays.

This study was supported by a grant to Dr. Victor from the Baxter Extramural Grant Program and in part by National Institutes of Health grant HL-31713 from the Specialized Center of Research in Hypertension (B1). Dr. Converse is supported by National Institutes of Health Career Development Grant No. 5-T32-HL07360-13. Dr. Jacobsen is supported by grants from The Danish Heart Foundation, Danish Research Academy, S\&W Foundation (Denmark), and Leo's Kemiske Fabrik Research Foundation (Denmark) and is a recipient of the Fogarty International Research Fellowship (NIH 1-F05-TW04494-01). Dr. Victor is supported by the National Heart, Lung, and Blood Institute (RO-1 HL44010) and is an Established Investigator of the American Heart Association.

\section{References}

1. Maher, J. F., and G. E. Schreiner. 1965. Hazards and complications of dialysis. N. Engl. J. Med. 273:370-377.

2. Henderson, L. W. 1980. Symptomatic hypotension during dialysis. Kidney Int. 17:571-576.

3. Henrich, W. L. 1986. Hemodynamic instability during hemodialysis. Kidney Int. 30:605-612.

4. Rostand, S. G., J. D. Brunzell, R. O. Cannon III, and R. G. Victor. 1992 Cardiovascular complications in renal failure. J. Am. Soc. Nephrol. 2:1053-1062.

5. Kersh, E. S., S. J. Kronfield, A. Unger, R. W. Popper, S. Cantor, and K. Cohn. 1974. Autonomic insufficiency in uremia as a cause of hemodialysis-induced hypotension. N. Engl. J. Med. 290:650-653.

6. Lilly, J. J., J. Golden, and R. A. Stone. 1976. Adrenergic regulation of blood pressure in chronic renal failure. J. Clin. Invest. 57:1190-1200.

7. McLeod, J. G. 1988. Autonomic dysfunction in peripheral nerve disease. In Autonomic Failure. R. Bannister editor. Oxford University Press, London. 615616.

8. Zoccali, C., M. Ciccarelli, and Q. Maggiore. 1982. Defective reflex control of heart rate in dialysis patients: evidence for an afferent autonomic lesion. Clin. Sci. 63:285-292.

9. Lazarus, J. M., C. L. Hampers, E. G. Lowrie, and J. P. Merrill. 1973. Baroreceptor activity in normotensive and hypertensive uremic patients. Circulation. 47:1015-1021.

10. Nies, A. S., D. Robertson, and W. J. Stone. 1979. Hemodialysis hypotension is not the result of uremic peripheral autonomic neuropathy. J. Lab. Clin. Med. 94:395-402.

11. Nakashima, Y., F. M. Fouad, S. Nakamoto, S. C. Textor, E. Bravo, and R. C. Tarazi. 1987. Localization of autonomic nervous system dysfunction in dialysis patients. Am. J. Nephrol. 7:375-381.

12. Mark, A. L., and G. Mancia. 1983. Arterial baroreflexes in humans. In Handbook of Physiology, Section 2: The Cardiovascular System, Volume III. J. T. Shepherd and F. M. Abboud, editors. American Physiological Society, Bethesda, MD. 755-785.

13. Skoog, P., J. Mansson, and P. Thorén. 1985. Changes in renal sympathetic outflow during hypotensive hemorrhage in rats. Acta Physiol. Scand. 125:655660 .

14. Victor, R. G., P. Thorén, D. A. Morgan, and A. L. Mark. 1989. Differential control of adrenal and renal sympathetic nerve activity during hemorrhagic hypotension in rats. Circ. Res. 64:686-694.

15. Morita, H., and S. F. Vatner. 1985. Effects of hemorrhage on renal nerve activity in conscious dogs. Circ. Res. 57:788-793.
16. Vallbo, A. B., K.-E. Hagbarth, H. E. Torebjörk, and B. G. Wallin. 1979. Somatosensory, proprioceptive, and sympathetic activity in human peripheral nerves. Physiol. Rev. 59:919-957.

17. Mark, A. L., R. G. Victor, C. Nerhed, and B. G. Wallin. 1985. Microneurographic studies of the mechanisms of sympathetic nerve responses to static exercise in humans. Circ. Res. 57:461-469.

18. Siggaard-Andersen, J. 1970. Venous occlusion plethysmography on the calf: Evaluation of diagnosis and results in vascular surgery. Dan. Med. Bull. 17(Suppl.):1-42.

19. Schiller, N. B., P. M. Shah, M. Crawford, A. DeMaria, R. Devereux, H. Feigenbaum, H. Gutgesell, N. Reichek, D. Sahn, I. Schnittger, et al. 1989. Recommendations for quantitation of the left ventricle by two-dimensional echocardiography. J. Am. Soc. Echocardiogr. 2:358-367.

20. Zoller, R. P., A. L. Mark, F. M. Abboud, P. G. Schmid, and D. D. Heistad. 1972. The role of low pressure baroreceptors in reflex vasoconstrictor responses in man. J. Clin. Invest. 51:2967-2972.

21. Callaghan, N. 1966. Restless legs syndrome in uremic neuropathy. Neurology. 16:359-361.

22. Jung, R., and M. D. Thames. 1991. Cardio-respiratory baroreflex control in rats with chronic myocardial infarction. Circulation. 84:II-554. (Abstr.)

23. Fagius, J., B. G. Wallin, G. Sundlöf, C. Nerhed, and S. Englesson. 1985. Sympathetic outflow in man after anaesthesia of the glossopharyngeal and vagus nerves. Brain. 108:423-438.

24. Aksamit, T. R., J. S. Floras, R. G. Victor, and P. E. Aylward. 1987. Paroxysmal hypertension due to sinoaortic baroreceptor denervation in humans. Hypertension. 9:309-314.

25. Robertson, D., G. A. Johnson, R. M. Robertson, A. S. Nies, D. G. Shand and J. A. Oates. 1979. Comparative assessment of stimuli that release neuronal and adrenomedullary catecholamines in man. Circulation. 59:637-643.

26. Brown, A. M. 1980. Receptors under pressure: an update on baroreceptors. Circ. Res. 46:1-10.

27. Secher, N. H., and P. Bie. 1985. Bradycardia during reversible hemorrhagic shock-a forgotten observation? Clin. Physiol. 5:315-323.

28. Almquist, A., I. F. Goldenberg, S. Milstein, M.-Y. Chen, X. Chen, R. Hansen, C. C. Gornick, and D. G. Benditt. 1989. Provocation of bradycardia and hypotension by isoproterenol and upright posture in patients with unexplained syncope. N. Engl. J. Med. 320:346-351.

29. Wallin, B. G., and G. Sundlöf. 1982. Sympathetic outflow to muscles during vasovagal syncope. J. Auton. Nerv. Syst. 6:287-291.

30. Scherrer U., S. F. Vissing, B. J. Morgan, P. Hanson, and R. G. Victor. 1990. Vasovagal syncope after infusion of a vasodilator in a heart-transplan recipient. N. Engl. J. Med. 322:602-604.

31. Lewis, T. 1932. Vasovagal syncope and the carotid sinus mechanism with comments on Gowers's and Nothnagel's syndrome. Br. Med. J. 1:873-876.

32. Schadt, J. C., M. D. McKown, D. P. McKown, and D. Franklin. 1984. Hemodynamic effects of hemorrhage and subsequent naloxone treatment in conscious rabbits. Am. J. Physiol. 247:R497-R508.

33. Morita, H., Y. Nishida, H. Motochigawa, N. Uemura, H. Hosomi, and S. F. Vatner. 1988. Opiate receptor-mediated decrease in renal nerve activity during hypotensive hemorrhage in conscious rabbits. Circ. Res. 63:165-172.

34. Bernton, E. W. 1985. Naloxone and TRH in the treatment of shock and trauma: What future roles? Ann. Emerg. Med. 14:729-735.

35. Smith, M. L., M. D. Carlson, H. M. Sheehan, and M. D. Thames. 1991. Naloxone does not prevent vasovagal syncope during simulated orthostasis in humans. Physiologist. 34:A27.2. (Abstr.)

36. Öberg, B., and P. Thorén. 1972. Increased activity in left ventricular receptors during hemorrhage or occlusion of caval veins in the cat: a possible cause of the vaso-vagal reactions. Acta Physiol. Scand. 85:164-173.

37. Abboud, F. M. 1989. Ventricular syncope: is the heart a sensory organ? $N$ Engl. J. Med. 320:390-392.

38. Lindner, A., B. Charra, D. J. Sherrard, and B. H. Scribner. 1974. Accelerated atherosclerosis in prolonged maintenance hemodialysis. N. Engl. J. Med. 290:697-701.

39. Thames, M. D., and F. M. Abboud. 1979. Reflex inhibition of renal sympathetic nerve activity during myocardial ischemia mediated by left ventricular receptors with vagal afferents in dogs. J. Clin. Invest. 63:395-402.

40. Nixon, J. V., J. H. Mitchell, J. J. McPhaul, Jr., and W. L. Henrich. 1983. Effects of hemodialysis on left ventricular function: dissociation of changes in filling volume and in contractile state. J. Clin. Invest. 71:377-384.

41. Morgan, D. A., P. Thorén, E. A. Wilczynski, R. G. Victor, and A. L. Mark. 1988. Serotonergic mechanisms mediate renal sympathoinhibition during severe hemorrhage in rats. Am. J. Physiol. 255:H496-H502.

42. Peuler, J. D., P. G. Schmid, D. A. Morgan, and A. L. Mark. 1990. Inhibition of renal sympathetic activity and heart rate by vasopressin in hemorrhaged diabetes insipidus rats. Am. J. Physiol. 258:H706-H712.

43. Landgren, S. 1952. On the excitation mechanism of the carotid baroreceptors. Acta Physiol. Scand. 26:1-34. 